\title{
Synthetic Turbulence: A Wavelet Based Simulation
}

Chao Yin ${ }^{\mathrm{a}}$, Teng $\mathrm{Wu}^{\mathrm{b}}$, Ahsan Kareem ${ }^{\mathrm{a}}$

${ }^{a}$ NatHaz Modeling Laboratory, Department of Civil and Environmental Engineering and Earth Sciences, University of Notre Dame, Notre Dame, IN 46556, USA

${ }^{b}$ Department of Civil, Structural and Environmental Engineering, University at Buffalo, State University of New York, Buffalo, NY 14260, USA; Corresponding author. Tel.: +1-716-6455152; fax: +1-716-645-3733, E-mail address: tengwu@buffalo.edu

Abstract: The treatment of wind-induced vibrations is an important consideration in the design of civil structures with increasing span-lengths and heights. The turbulence in the atmospheric boundary layer has been treated as Gaussian in conventional stochastic simulation schemes, wherein higher-order statistics have been disregarded. However, experimental evidence points at turbulence being a typical multifractal process, which suggests that the statistics at different scales of atmospheric turbulence are not strictly selfsimilar but exhibit stronger non-Gaussianity as the length scale decreases. Intermittency characterized by the occasional bursts in the wind velocity leads to non-Gaussianity. Intermittency and its potential impact on wind-induced response have been neglected though. Recent studies have addressed the multifractal property of turbulence in wind with wavelet-basis representations of the log-Normal or log-Poisson models. These schemes offer new insight into the simulation of turbulent wind, but suffer from several significant drawbacks, such as the inappropriate sampling of the wavelet coefficients. To overcome these shortcomings, a new simulation scheme, based on the Haar wavelet representation, 
23 is proposed in this study where the exact relation between the wind velocities and the

24 wavelet coefficients is introduced. In addition, the effects of intermittency on the wind-

25 induced response of structures are evaluated for a number of cases. A quasi-steady theory-

26 based assessment indicates that the intermittency results in amplifying extremes of the

27 wind-induced response and exhibits higher impact on relatively more rigid structures. It is

28 likely that intermittency may invoke flow-structure interaction with possible enhancement

29 in load effects at related scales, which may further amplify the extremes.

30 Keywords: Stochastic simulation; turbulent flow; wind process; intermittency; log-Poisson 31 model; wavelet.

\section{INTRODUCTION}

34 Civil structures, such as bridges, buildings, transmission towers and residential structures, 35 are vulnerable to the winds and the associated turbulence. Turbulence has attracted a wide 36 range of studies including the chaotic features, which imply high level of nonlinearity and 37 unpredictability. In general, there are two distinct approaches to describe a turbulent flow: 38 one developed from first principles using the equations of motion, e.g. Navier-Stokes 39 equations, or the probabilistic framework utilizing statistics of turbulent flows based on 40 observations.

$41 \quad$ Numerical simulation of turbulent wind field using the Navier-Stokes equations is 42 computationally very intensive, especially at high Reynold numbers, whereas statistical 43 approaches can significantly reduce the computational effort by utilizing turbulence 44 statistics. Conventionally, turbulence in the wind field is characterized by the second-order 45 statistics. For example, both the spectral representation and the time series models are 
46 based on the second-order statistics with implied assumption of Gaussianity (Shinozuka

47 and Deodatis 1991; Li and Kareem 1990a; 1990b). However, turbulence has significant

48 higher-order information, which is usually described in terms of velocity increment

49 between two time instants. Experiments have shown that the statistics of the velocity

50 increment depart gradually away from Gaussianity as the time interval decreases (Atta and

51 Chen 1970; Castaing et al. 1990; Frisch 1995). The K41 theory of turbulence divides the

52 velocity increment into energy-containing, inertial and dissipation sub-ranges

53 (Kolmogorov 1941). The probability density functions (PDFs) of velocity increments in

54 the inertial sub-range present longer tails than the Gaussian distribution. The non-

55 Gaussianity is highly correlated with the intermittency in turbulent flows. Theoretically,

56 non-Gaussianity features of the PDF reflect the presence of intermittency in the velocity

57 increments and gradients (e.g., Sreenivasan and Antonia 1997). The intermittency has

58 potential effects on wind-induced response because it occurs suddenly with attendant high

59 energy, which may be underestimated by a tacit Gaussian assumption. The higher-order

60 statistics of the wind velocity process can be approximately derived according to their

61 relation to the second-order statistics. For example, both the log-Normal model

62 (Kolmogorov 1962) and the log-Poisson model (She and Leveque 1994) show high-fidelity

63 for the derivation of higher-order statistics. The log-Poisson model is shown to perform

64 better in She and Waymire (1995), and thus it has been used in the present simulation

65 scheme. Indeed, both the log-Normal model and the log-Poisson model introduce a

66 multifractal description of wind fluctuations, rendering the simulation process non-trivial.

67 In this context, a wavelet expansion, which is a multi-scale modeling tool, is a preferred

68 basis (e.g., Arneodo et al. 1998; Gurley and Kareem 1999; Kitagawa and Nomura 2003). 
69 In this approach, the sampling of wavelet coefficients from the multifractal statistics is

70 crucial to a high-fidelity simulation of the wind field. In these references initial work in

71 this context was introduced which is further explored and refined here.

This study proposes a new scheme for simulating wind velocity based on the Haar

73 wavelet. This entails several assumptions, i.e., (1) the wind velocity increment in the

74 energy-containing sub-range is only affected by the boundary conditions; (2) statistics of

75 the wind velocity increment in the inertial sub-range follow a log-Poisson model for both

76 stationary and non-stationary winds; (3) the wind velocity increment in the energy-

77 dissipation sub-range has negligible contribution to wind fluctuations with practical

78 implications, and is therefore disregarded. The second assumption relates to the

79 Kolmogorov's conjecture that within a small time interval the statistics of velocity

80 increment are approximately steady even when the wind velocity is not (Kolmogorov

81 1941).

82 The higher-order effects in velocity increments related to intermittency are 83 highlighted here. Typically, the wind-induced response is based on the gust loading factor 84 which involves a peak factor derived under Gaussian assumption. Three major issues, 85 which have been usually ignored, are taken into account in the study to examine their 86 contribution: the quadratic velocity term; the inclusion of relative motion in the formulation 87 of aerodynamic force; the higher-order statistics of velocity fluctuations.

\section{DESCRIPTION OF THE WIND VELOCITY PROCESS}

89 A wind velocity as a random process can be expressed in terms of the Karhunen-Loeve (K-

90 L) expansion as 
92 where $V(\boldsymbol{\theta}, t)$ represents a wind velocity indexed by time $t ; \boldsymbol{\theta}$ is the vector of the underlying

93 random parameters of the wind velocity process; $\left\{\xi_{j}(\boldsymbol{\theta})\right\}_{j=0}^{N}$ denotes a normalized 94 decomposed random vector with a scaling vector $\left\{\lambda_{j}\right\}_{j=0}^{N}$, which also represents the 95 eigenvalue vector of K-L expansion; $\left\{\varphi_{j}(\boldsymbol{\theta}, t)\right\}_{j=0}^{N}$ is the corresponding basis.

The Fourier basis is often used in the simulation of a stationary process, whereas a

97 wavelet basis is equally applicable to both stationary and non-stationary processes. Both

98 the spectral representation and the time series (e.g., ARMA) models imply stationarity with

99 the assumption that $\left\{\xi_{j}(\boldsymbol{\theta})\right\}_{j=0}^{N}$ is a Gaussian random vector (Shinozuka and Deodatis

100 1991; Li and Kareem 1990a; 1990b). The basis $\left\{\varphi_{j}(\boldsymbol{\theta}, t)\right\}_{j=0}^{N}$ can be further expanded in

101 terms of a wavelet basis as (e.g., Ghanem and Spanos 2003; Phoon et al. 2004)

102

$\varphi_{j}(\boldsymbol{\theta}, t)=\sum_{k=1}^{M} \zeta_{j, k}(\boldsymbol{\theta}) \psi_{j, k}(t)$ for $j=0, \ldots, N$

103 where $\left\{\psi_{j, k}(t)\right\}_{j=0, k=1}^{N, M}$ is the wavelet basis; $\left\{\zeta_{j, k}(\boldsymbol{\theta})\right\}_{k=1}^{M}$ is the Gaussian random vector.

104 As a result, the wind velocity can be further expressed as

105

$$
\begin{aligned}
V(\boldsymbol{\theta}, t) & =\sum_{j=0}^{N}\left\{\xi_{j}(\boldsymbol{\theta}) \lambda_{j} \sum_{k=1}^{M} \tilde{\zeta}_{j, k}(\boldsymbol{\theta}) \psi_{j, k}(t)\right\} \\
& =\sum_{j=0}^{N} \sum_{k=1}^{M} \zeta_{j, k}(\boldsymbol{\theta}) m_{j, k} \psi_{j, k}(t)
\end{aligned}
$$

106 where $\left\{\zeta_{j, k}(\boldsymbol{\theta})\right\}_{k=1}^{M}$ denotes a normalized Gaussian random vector with a scaling vector $107\left\{m_{j, k}\right\}_{k=1}^{M}$, which also represents the vector of wavelet coefficients. 
109 power law (Bak et al. 1988). For example, the power spectrum of a typical turbulent wind

110 has the relation $S(f) \sim f^{5 / 3}$ in the inertial range (Kolmogorov 1941). The power law implies

111 the existence of self-similarity or scale-invariance in turbulence (Bak et al. 1988), i.e., the

112 PDFs of velocity increments at different length or time scales are similar. Here the velocity

113 increment is defined as $\delta V=V(t)-V(t-\tau)$, in which $\tau$ is a time interval. However, further

114 investigation of turbulence structure has shown that the PDFs at different scales are not

115 strictly similar but gradually depart from Gaussian density functions as the length scale

116 decreases. This scale-dependent or multifractal property implies that the turbulent wind is

117 not a Gaussian process (e.g., Basu et al. 2004). Figure 1 presents the PDFs of velocity

118 increments in various time intervals for the comparison of a measured turbulent wind

119 velocity and the corresponding spectral representation. It shows that, the spectral

120 representation naturally leads to the PDFs at different time intervals to be Gaussian,

121 whereas for the measured turbulent wind, the PDFs present tails longer than the Gaussian.

122 The longer tails in the PDFs are a reflection of the intermittency phenomenon of turbulence.

123 The statistical explanation refers to the theory of large-deviation (Frisch 1995). Due to the

124 multifractal nature of turbulence, a multi-scale simulation framework like the wavelet

125 representation would be a more appropriate representation of wind velocity fluctuations,

126 which could be utilized for numerical simulation.

\section{$127 \quad 2.1$ Multifractal Property of Turbulence}

128 The inertial range of turbulent wind is characterized by two length scales, $l_{c}$ and $l_{d}$, which 129 represent the smallest scales of the energy-containing range and inertial range, respectively. 130 The multifractal property of turbulence can be quantified following two basic parameters, 
131 the energy dissipation rate $\varepsilon_{l}$ and the velocity increment $\delta V_{l}$. Here $\delta V_{l}$ denotes the velocity

132 increment with time interval $l$. For the velocity increment within the inertial range, the $p$ th

133 order moments of $\delta V_{l}$ and $\varepsilon l$ are both dependent on $l$ (She and Waymire 1995)

134

$\left\langle\delta V_{l}^{p}\right\rangle \sim l^{\alpha_{p}}$

$135\left\langle\varepsilon_{l}^{p}\right\rangle \sim l^{\tau_{p}}$

136 and

$137 \quad \alpha_{p}=\frac{p}{3}+\frac{\tau_{p}}{3}$

138 where $\langle\cdot\rangle$ denotes the expectation operator; $\tau_{p}$ and $\alpha_{p}$ are both the exponents related to the $139 p$ th order moment.

\section{$140 \quad 2.2$ A Hierarchical Relation of Velocity Increments}

141 In K41 theory, $\tau_{p}=0$, implying that the statistics of $\varepsilon$ is scale-invariant. However, for a

142 measured turbulence, the statistics of $\varepsilon$ are actually scale-dependent $\left(\tau_{p} \neq 0\right)$. Various

143 models have been proposed to model the statistics of $\delta v$ and $\varepsilon$ through the modification of

$144 \tau_{p}$ (Kolmogorov 1962; She and Leveque 1994). Among them one of the most promising

145 models may be the log-Poisson model (She and Leveque 1994; She and Waymire 1995).

146 Let $l_{j}$ denote the length of the $j$ th scale. As $j$ decreases, the scale becomes larger. The log-

147 Poisson model suggests that, within the inertial range, the velocity increments $\delta V_{l_{1}}$ and

$148 \delta V_{l_{2}}$ have the following hierarchical relation (She and Waymire 1995)

$149 \quad \delta V_{l_{2}}=W_{l_{1} l_{2}} \delta V_{l_{1}}=\left[\left(\frac{l_{1}}{l_{2}}\right)^{\gamma-1} \beta^{\kappa_{l_{1} l_{2}}}\right]^{\frac{1}{3}} \delta V_{l_{1}}$ 
150 where $W_{l_{1} l_{2}}$ is a multiplicative factor from $\delta V_{l_{1}}$ to $\delta V_{l_{2}} ; \gamma$ and $\beta$ are scale-related 151 parameters estimated from the real turbulent wind. In this study, the values are set to $\gamma=$ $1522 / 3$ and $\beta=2 / 3$ as suggested by She and Waymire (1995). $\kappa_{l_{1} l_{2}}$ is a Poisson random 153 variable with mean $\lambda_{l_{1} l_{2}}$, which satisfies the following relation

$154 \quad \lambda_{l_{1} l_{2}}=-\frac{\gamma}{\beta-1} \ln \frac{l_{1}}{l_{2}}$

155 It should be noted that the hierarchical relation from scale $l_{1}$ to $l_{3}$ is identical to the relation 156 from scale $l_{1}$ to $l_{3}$ via an immediate scale $l_{2}$, i.e.,

$157 \delta V_{l_{3}}=W_{l_{2} l_{3}} W_{l_{1} l_{2}} \delta V_{l_{1}}=W_{l_{1} l_{3}} \delta V_{l_{1}}$

\section{SYNTHETIC TURBULENCE USING A WAVELET} 159 REPRESENTATION

\subsection{Wavelet Representation}

161 Equation (3) suggests that a general random process can be represented in the wavelet 162 domain. Here an orthogonal wavelet basis is employed for efficiency. Thus the wind 163 velocity process can be further expressed as

$$
V(\boldsymbol{\theta}, t)=\zeta_{\phi}(\boldsymbol{\theta}) m_{\phi} \phi(t)+\sum_{j=1}^{n} \sum_{k=1}^{2^{j-1}} \zeta_{j, k}(\boldsymbol{\theta}) m_{j, k} \psi_{j, k}(t)
$$

165 where $\zeta_{\phi}(\boldsymbol{\theta})$ is the constant $1 ; m_{\phi}$ represents the wavelet coefficient corresponding to the 166 father wavelet $\phi(t)$; the definition of $\left\{\zeta_{j, k}(\boldsymbol{\theta})\right\}_{j=1, k=1}^{n, 2^{j-1}}$ is in Section 3.3; $m_{j, k}$ represents the 
167 wavelet coefficient at the $j$ th scale and $k$ th time position corresponding to the wavelet $168 \psi_{j, k}(t)$, which is defined as:

$169 \psi_{j, k}(t)=2^{-(n-j+1) / 2} \psi\left(\frac{t-2^{n-j+1}(k-1)}{2^{n-j+1}}\right) \quad$ for $\quad 1 \leq j \leq n, 1 \leq k \leq 2^{j-1}$

170 in which $\psi(t)$ is the mother wavelet. It should be noted that, for the purpose of relating it

171 to the scale order in turbulence, the scale order in the above equation is opposite to the

172 common definition used in the wavelet analysis.

\section{$173 \quad 3.2$ Selection of Wavelet Basis}

174 An appropriate wavelet basis should establish a connection between the velocity 175 increments and the wavelet coefficients. For example, the Haar wavelet basis has the 176 following father and mother wavelets, respectively

$177 \phi(x)= \begin{cases}1 & 0<x \leq 1 \\ 0 & \text { otherwise }\end{cases}$

$178 \psi(x)= \begin{cases}1 & 0<x \leq \frac{1}{2} \\ -1 & \frac{1}{2}<x \leq 1 \\ 0 & \text { otherwise }\end{cases}$

179 as shown in Fig. 2(a). The wavelet coefficient at the smallest scale $(j=n)$ can be calculated 180 as

181

$$
\begin{aligned}
m_{n, k} & =\int_{-\infty}^{\infty} v(t) \psi_{n, k}(t) d t \\
& =\frac{1}{\sqrt{2}}[v(2 k-1)-v(2 k)] \quad \text { for } \quad k=1, \ldots, 2^{n-1}
\end{aligned}
$$


182 which coincides with the velocity increment in the time interval 1 scaled by $\frac{1}{\sqrt{2}}$. In general,

$$
\begin{aligned}
m_{j, k} & =\int_{-\infty}^{\infty} v(t) \psi_{j, k}(t) d t \\
& =\frac{1}{2^{(n-j+1) / 2}} \sum_{i=2^{n-j} \times(k-1)+1}^{2^{n-j} \times k}\left[v(i)-v\left(i+2^{n-j}\right)\right] \quad \text { for } \quad k=1, \ldots, 2^{j-1}
\end{aligned}
$$

which represents the sum of several velocity increments in the time interval $2^{n-j}$. Here $\delta v_{l_{j}, i}$

185 is used to denote the velocity increment at time instant $i$ with time interval $l_{j}=2^{n-j}$. Thus, 186 according to Eq. (15), there is

$187 \quad m_{j, k}=\frac{1}{2^{(n-j+1) / 2}} \sum_{i=2^{n-j} \times(k-1)+1}^{2^{n-j} \times k} \delta v_{l_{j}, i}$

188 The above equations demonstrate that the Haar wavelet coefficients can be exactly derived

189 from the velocity increments at the same scale. A simple example is provided here as an

190 illustration. Suppose there is a wind velocity process $v(t)$ indexed by 8 time instants as 191 follows

1. The smallest scale:

$$
\begin{aligned}
& m_{3,1}=\frac{1}{\sqrt{2}} \delta v_{l_{3}, 1}=\frac{1}{\sqrt{2}}\left(v_{1}-v_{2}\right) \\
& m_{3,2}=\frac{1}{\sqrt{2}} \delta v_{l_{3}, 3}=\frac{1}{\sqrt{2}}\left(v_{3}-v_{4}\right) \\
& m_{3,3}=\frac{1}{\sqrt{2}} \delta v_{l_{3}, 5}=\frac{1}{\sqrt{2}}\left(v_{5}-v_{6}\right) \\
& m_{3,4}=\frac{1}{\sqrt{2}} \delta v_{l_{3}, 7}=\frac{1}{\sqrt{2}}\left(v_{7}-v_{8}\right)
\end{aligned}
$$

2. The intermediate scale: 


$$
\begin{aligned}
& m_{2,1}=\frac{1}{2} \sum_{i=1}^{2} \delta v_{l_{2}, i}=\frac{1}{2}\left[\left(v_{1}-v_{3}\right)+\left(v_{2}-v_{4}\right)\right] \\
& m_{2,2}=\frac{1}{2} \sum_{i=3}^{4} \delta v_{l_{2}, i}=\frac{1}{2}\left[\left(v_{5}-v_{7}\right)+\left(v_{6}-v_{8}\right)\right]
\end{aligned}
$$

3. The largest scale:

$$
\begin{aligned}
m_{1,1} & =\frac{1}{2 \sqrt{2}} \sum_{i=1}^{4} \delta v_{l_{1}, i} \\
& =\frac{1}{2 \sqrt{2}}\left[\left(v_{1}-v_{5}\right)+\left(v_{2}-v_{6}\right)+\left(v_{3}-v_{7}\right)+\left(v_{4}-v_{8}\right)\right]
\end{aligned}
$$

198 Relations between the Haar wavelet coefficients and velocity increments are detailed in 199 Fig. 3.

202 wavelet, as shown in Fig. 2(b), requires that the calculation of its corresponding wavelet

203 coefficients should involve the velocity increments in different time intervals. As a 204 consequence, the statistics of the wavelet coefficients are not conveniently derived from 205 the velocity increments.

\section{$206 \quad 3.3$ Simulation of Turbulent Wind}

207 As mentioned previously, turbulent wind consists of energy-containing, inertial and 208 dissipation sub-ranges. A critical length $l_{c}$ separates the energy-containing sub-range from 209 the inertial sub-range. In this study, $l_{c}$ is assumed corresponding to the critical frequency $f_{c}$ 210 which makes $f \hat{S}_{V}(f)$ maximum, where $\hat{S}_{V}(f)$ is the power spectrum of the zero mean 211 wind velocity process $V(t)$ (Kitagawa and Nomura 2003), i.e., 
$212 f_{c}=\arg \max _{f \in(0, \infty)} f \widehat{S}_{V}(f)$

214 relation to $f_{c}$ as follows

$215 j_{c}=n+\operatorname{int}\left\{\log _{2}\left(\frac{f_{c}}{0.742}\right)\right\}$

216 Details are shown in Appendix A.

217 Let $\delta U_{l_{j}, i}$ be the normalized version of $\delta V_{l_{j}, i}$. The simulation procedure of the 218 turbulent wind based on the Haar wavelet is illustrated in Fig. 4, and interpreted in details 219 as follows

220 1. Determine $j_{c}$ by Eq. (21);

221 2. For $l \geq l_{c}$ or $j \leq j_{c}$, i.e., within the energy-containing sub-range, $\left\{\delta U_{l_{j}, i}\right\}_{i=1}^{2^{n-1}}$ is an 222 identical independent standard Gaussian sequence (Kitagawa and Nomura 2003), 223 and $\zeta_{j, k}(\boldsymbol{\theta})=\delta U_{l_{j}, 2^{n-j}(k-1)+1}$ for $k=1, \ldots, 2^{j-1}$; $\left\{\delta U_{l_{c}, i}\right\}_{i=1}^{2^{n-1}}$ with an identical independent multiplicative sequence $\left\{W_{j_{c} j}(i)\right\}_{i=1}^{2^{n-1}}$ as:

$$
\delta U_{l_{j}, i} \propto W_{j_{c} j}(i) \delta U_{l_{c}, i}
$$

where

228

$$
W_{j_{c} j}(i)=\left(\frac{l_{j_{c}}}{l_{j}}\right)^{\gamma} \beta^{\kappa_{j_{c} j}}(i)
$$


and $\left\{\kappa_{j_{c} j}(i)\right\}_{i=1}^{2^{n-1}}$ is an identical independent Poisson random sequence with mean

$$
\zeta_{j, k}(\boldsymbol{\theta}) \propto \frac{1}{2^{(n-j+1) / 2}} \sum_{i=2^{n-j} \times(k-1)+1}^{2^{n-j} \times k} \delta U_{l_{j}, i}
$$

4. $\left\{m_{j, k}\right\}_{j=1, k=1}^{n, 2^{j-1}}$ is determined by power spectrum as (see Appendix B)

$$
m_{j, k}=\sqrt{2^{n-j+1} \int_{-\infty}^{\infty}|\tilde{\psi}(f)|^{2} S_{V}(f) d f}
$$

where $\tilde{\psi}(f)$ is the Fourier spectrum of $\psi(t)$; $S_{V}(f)$ is the power spectrum without

$$
m_{\phi}=\sqrt{\int_{-\infty}^{\infty}|\tilde{\phi}(f)|^{2} S_{V}(f) d f}
$$

where $\tilde{\phi}(f)$ is the Fourier spectrum of $\phi(t)$. If the target turbulent wind is nonstationary, as noted in the introduction, $\left\{m_{j, k}\right\}_{j=1, k=1}^{j_{c}, 2^{j_{c}-1}}$ should be adjusted by matching the wavelet spectra.

5. The simulated turbulent wind is obtained according to Eq. (10).

243 stationary if the wind velocity process is stationary (see Appendix B). For a non-stationary 244 turbulent wind, the non-stationarity is assumed only related to the wavelet coefficients in 245 the energy-containing range, as mentioned in the second assumption.

\section{$246 \quad 3.4$ Simulation Example}


247 The wind velocity data recorded during Hurricane Katrina was fitted to the Karman

248 spectrum model to simulate the turbulence, where the turbulence integral scale was

249 estimated to be $247 \mathrm{~m}$ and $j_{c}=7$. The simulation results based on the proposed and the

250 spectral representation schemes are shown in Fig. 5. The Karman spectrum and the power

251 spectrum of the simulation are presented in Fig. 6. Also, PDFs of wavelet coefficients at

252 several typical scales in the inertial sub-range are given in Fig. 7. The results show that the

253 non-Gaussianity in turbulent wind is captured by the proposed simulation scheme.

\section{3.5 Comparison with Other Simulation Schemes in the Wavelet Domain}

255 It should be emphasized that, in the inertial sub-range, the statistics of the velocity 256 increments at various scales, where energy production and dissipation balance, are obtained 257 based on the stationarity assumption (She and Leveque 1994; She and Waymire 1995).

258 While the multi-scale system may not be in equilibrium at a particular time instant because 259 of the intermittency, the averaged statistics of the velocity increments at respective scales 260 may be stationary. The present simulation scheme is based on this premise and illustrated 261 in Fig. 8, where $\zeta_{j}$, represents a random variable that has the identical statistics with $\zeta_{j, 1}$, $262 \zeta_{j, 2}, \ldots, \zeta_{j, k}$. The statistics of $\zeta_{j_{c}+1}$, are derived from $\zeta_{j_{c}}$. based on the log-Poisson model. 263 Figure 8 illustrates that the wavelet coefficients at a particular scale in the inertial sub264 range are identical. The conditional relations of these wavelet coefficients to those at other 265 scales are also identical. Thus it may not be reasonable to discriminate the identical 266 conditional relations. The next two subsections will revisit two typical simulation schemes 267 based on wavelet representations and make remarks accordingly.

\subsubsection{Wavelet dyadic trees model}


269 The wavelet dyadic trees (WDT) model (Arneodo et al. 1998), as illustrated in Fig. 9, is

270 expressed as follows

$m_{0,1}=1$

$271 \quad m_{j, 2 k-1}=W_{j-1, k}^{l} m_{j-1, k}$

$m_{j, 2 k}=W_{j-1, k}^{r} m_{j-1, k} \quad$ for $\quad 1 \leq j \leq n, 1 \leq k \leq 2^{j-1}$

272 where $m_{j, k}$ is a wavelet coefficient; multiplicative factors $W_{j-1, k}^{l}$ and $W_{j-1, k}^{r}$ are identical

273 random variables and have the same statistics as defined in Eq. (7). Note that $m_{j, k}$ is not a

274 deterministic but a random variable here. Both $m_{j, 2 k-1}$ and $m_{j, 2 k}$ derive from the same father

275 variable $m_{j-1, k}$ and have identical statistics. However, the sequence $\left\{m_{j, k}\right\}_{k=1}^{2^{j-1}}$ is not

276 stationary because the correlation function between $m_{j, 2 k-1}$ and $m_{j, 2 k}$ is not equal to that

277 between $m_{j, 2 k}$ and $m_{j, 2 k+1}$. A brief explanation is that, for example, the correlation function

278 between $m_{2,1}$ and $m_{2,2}$ depends on the sampling of $W_{1,1}^{l}$ and $W_{1,1}^{r}$, while the correlation

279 function between $m_{2,2}$ and $m_{2,3}$ depends on the sampling of not only $W_{1,1}^{r}$ and $W_{1,2}^{l}$ but also

$280 W_{0,1}^{l}$ and $W_{0,1}^{r}$ at the higher level. $W_{0,1}^{l}$ and $W_{0,1}^{r}$ introduce additional influence on the

281 calculation of the correlation function between $m_{2,2}$ and $m_{2,3}$ in comparison with that

282 between $m_{2,1}$ and $m_{2,2}$, and hence causes nonstationarity of the wavelet coefficients at scale

$283 j=2$. A simple example here helps to further explain it. Suppose there are two random

284 variables, $Z_{1}$ and $Z_{2}$, which are respectively defined as

285

$Z_{1}=X+\sum_{i=1}^{20} Y_{i}=X+\sum_{i=1}^{20} W_{i} X$
$Z_{2}=X+\sum_{i=1}^{20} \tilde{Y}_{i}=X+\sum_{i=1}^{20} W_{i} \tilde{X}_{i}$ 
286 where $X$ is a standard Gaussian random variable; $\left\{W_{i}\right\}_{i=1}^{20}$ and $\left\{\tilde{X}_{i}\right\}_{i=1}^{20}$ are both identical 287 independent standard Gaussian random sequences. Although $Y_{i}$ has the identical statistics 288 with $\tilde{Y}_{i}, Y_{i}$ indeed depends on $X$ while $\tilde{Y}_{i}$ is independent of $X$. Therefore, to simulate $Z_{1}$, the 289 sampling of $\left\{Y_{i}\right\}_{i=1}^{20}$ is actually conditionally dependent on the sampling of $X$. Four 290 sampling pairs of $X$ and $\left\{Y_{i}\right\}_{i=1}^{20}$ are plotted in Fig. 10(a), which shows a strong correlation

291 between $X$ and $Y_{i}$. It may be imagined that the concatenated samplings of $\left\{Y_{i}\right\}_{i=1}^{20}$ are not 292 stationary. For comparison Fig. 10(b) plots four sampling pairs of $X$ and $\left\{\tilde{Y}_{i}\right\}_{i=1}^{20}$. Obviously, 293 the concatenated samplings of $\left\{\tilde{Y}_{i}\right\}_{i=1}^{20}$ approach stationary.

In addition to nonstationarity, WDT model does not present an exact relation 295 between the velocity increments and the wavelet coefficients.

\subsubsection{A modified WDT model}

297 A modified WDT model is expressed as follows (Kitagawa and Nomura 2003)

298

$\zeta_{j, 2 k-1}=S \times W_{j-1, k} \zeta_{j-1, k}$

$\zeta_{j, 2 k}=S \times W_{j-1, k} \frac{\sqrt{2}\left(\zeta_{j-1, k}+\zeta_{j-1, k+1}\right)}{2} \quad$ for $\quad j_{c}<j \leq n, 1 \leq k \leq 2^{j-1}$

299 where $\zeta_{j, k}$ is a normalized random variable; $W_{j, k}$ is a multiplicative factor as defined in Eq.

300 (7); $S$ is a binary random variable having two values: -1 and 1 . In particular, $\zeta_{j_{c}, k}$ is a 301 standard Gaussian random variable. The simulation scheme is illustrated in Fig. 11. In 302 order to alleviate the nonstationarity of the WDT model, the modified WDT model 303 introduces artificial correlation among wavelet coefficients derived from different fathers. 304 According to Eq. (29), since $\zeta_{j, 2 k-1}$ derives from only $\zeta_{j-1, k}$ while $\zeta_{j, 2 k}$ from both $\zeta_{j-1, k}$ 305 and $\zeta_{j-1, k+1}, \zeta_{j, 2 k-1}$ has different statistics from $\zeta_{j, 2 k}$. The artificial modification of $\zeta_{j, 2 k}$ 
306 introduces additional nonstationarity in $\left\{\zeta_{j, k}\right\}_{k=1}^{2^{j-1}}$. Besides, the exact relation between the 307 wavelet coefficients and the velocity increments is still absent in the modified WDT model.

308 In light of the preceding comparison, it is concluded that the Haar wavelet based scheme 309 presented earlier captures required features and reflects necessary correlation structures.

\section{ASSESSMENT OF INTERMITTENCY EFFECTS}

311 A simple example of a single degree of freedom (SDOF) structure is utilized for the 312 assessment of the intermittency effects on the alongwind-induced response of structures 313 based on quasi-steady aerodynamics. The wind-induced response is described by

$314 m \ddot{X}(t)+c \dot{X}(t)+k X(t)=\frac{1}{2} \rho A C_{D}[\bar{V}+V(t)-\dot{X}(t)]^{2}$

315 where $m, c$ and $k$ represent respectively the mass, damping coefficient and stiffness of the 316 structure; $\ddot{X}(t), \dot{X}(t)$ and $X(t)$ represent respectively the acceleration, velocity and 317 displacement of the structure; $\rho$ is the air density; $A$ is the projection area of the wind load; $318 C_{D}$ is the drag coefficient; $\bar{V}$ is the mean wind velocity and $V(t)$ is the fluctuating velocity.

319 4.1 Non-Gaussian Structural Response Peak Factor

320 Rewrite Eq. (30) as

321

$$
m \ddot{X}(t)+\left\{c+\rho A C_{D}[\bar{V}+V(t)]\right\} \dot{X}(t)+k X(t)=\frac{1}{2} \rho A C_{D}\left\{[\bar{V}+V(t)]^{2}+[\dot{X}(t)]^{2}\right\}
$$

322 As the above equation indicates, the transformation from wind velocity to wind load, $323[\bar{V}+V(t)]^{2}$, involves a quadratic effect that leads to a non-Gaussianity. The interaction 324 between the structural motion and wind forces gives rise to a time-variant damping 
325 coefficient $\rho A C_{D}[\bar{V}+V(t)]$ and a feedback force $\frac{1}{2} \rho A C_{D} \dot{X}^{2}(t)$, which enhance the non326 Gaussianity of the response process $X(t)$. As a consequence, the marginal PDF of $X(t)$ may 327 have a longer tail than the corresponding Gaussian density function. The longer tail (or 328 large deviation) has a significant impact on the extreme value $Y(t)=\max (|X(s)|: s \in[0$, $329 t]$ ). The quadratic and interaction effects have been well examined under the assumption of 330 the Gaussian wind velocity fluctuation (e.g., Grigoriu 1986; Kareem et al. 1998). These 331 studies suggest that if the two added effects were disregarded, the extreme value of $X(t)$ 332 would be underestimated. However, since $V(t)$ is strictly not a Gaussian process, it may 333 further impact the extremes. Here the effects of non-Gaussianity caused by the 334 intermittency in $V(t)$ on extremes of a SDOF system is evaluated. 336 peak factor, which is defined as

$337 \quad g(t)=\frac{\langle Y(t)\rangle-\langle X(t)\rangle}{\sigma_{X}(t)}$

338 where $g(t)$ is the structural response peak factor; $\sigma_{X}(t)$ represents the stand deviation of the 339 structural displacement. The PDF of $Y(t)$ can be expressed as an exponential form (Lutes 340 and Sarkani 1997)

341

$$
p_{Y}(y, t)=\frac{\partial P_{Y}(y, t)}{\partial y}=\frac{\partial\left[P_{Y}(y, 0) \exp \left(-\int_{0}^{t} \eta_{X}(y, s) d s\right)\right]}{\partial y}
$$

342 where $P_{Y}(y, t)$ denotes the cumulative distribution function of $Y(t) ; \eta x(y, s)$ is a hazard 343 function in reliability theory. Based on the assumption of a Poisson process, it is assumed 344 to be equal to the zero crossing rate (Lutes and Sarkani 1997) 


$$
\begin{aligned}
\eta_{X}(y, s) & \approx v_{X}(y, s) \\
& =\int_{0}^{\infty} \dot{x} p_{X \dot{x}}(y, \dot{x} \mid X(0) \leq y, \text { no upcrossings in }[0, s]) d \dot{x}
\end{aligned}
$$

346 Further assumptions are necessary due to the complexity in the computation of the 347 conditional joint PDF $p_{X \dot{X}}$. If $X(t)$ is covariance stationary, $X(t)$ and $\dot{X}(t)$ should be 348 uncorrelated; if $X(t)$ is a Gaussian stationary process, $X(t)$ and $\dot{X}(t)$ should be mutually 349 independent. The structural response peak factor is conventionally calculated based on the 350 assumption that the structural response process is a Gaussian stationary process (Davenport 351 1964). An improved relationship takes into account the non-Gaussian property of $X(t)$ (e.g., 352 Grigoriu 1986; Kareem et al. 1998). In this study, the Monte Carlo simulation (MCS) is 353 employed in the computation of the intermittency effects. The sampling scheme for $g(t)$ is

$354 \quad g(t) \approx \frac{\sum_{i=1}^{N}\left[y\left(\boldsymbol{\theta}_{i}, t\right)-x\left(\boldsymbol{\theta}_{i}, t\right)\right]}{\sqrt{\sum_{i=1}^{N} x^{2}\left(\boldsymbol{\theta}_{i}, t\right)-\left[\sum_{i=1}^{N} x\left(\boldsymbol{\theta}_{i}, t\right)\right]^{2}}}$

355 where $N$ represents the number of samples. Each sample $x\left(\boldsymbol{\theta}_{i}, t\right)$ is calculated by the 356 Newmark- $\beta$ method.

\section{$357 \quad 4.2$ Case Study}

358 Consider the SDOF system as a water tower used in Grigoriu (1986). Seven cases, with 359 various combinations of the wind profile, structural mass and damping ratio, are studied. 360 The incident wind velocity for the SDOF system is determined by its height, the reference 361 wind velocity and the power law exponent of the wind profile. The results are listed in 362 Table 1, in which $h, M$ and $K$ represent respectively the height, mass and stiffness of the 
363 water tower. The auto-covariance function of the wind velocity process is given as

364 (Grigoriu 1986)

$365 \quad R_{v}(\tau)=\sigma_{v}^{2} \sum_{i=1}^{2} p_{i} e^{-q_{i}|\tau|}$

366 in which $p_{1}=0.492365 ; p_{2}=1-p_{1} ; q_{1}=0.0646863 ; q_{2}=0.28891$. The time step chosen in

367 the Monte Carlo simulation is 0.1 seconds, which ensures that all structural frequencies are

368 captured. The duration time is $2^{15} \times 0.1=3276.8$ seconds.

369 The following observations are offered based on the results:

370 1. Under the assumption of Gaussian wind velocity process, the structural response 371 peak factors calculated by the MCS are generally smaller than the closed-form 372 solutions (Grigoriu 1986; Kareem et al. 1998). A possible reason is that, the closed373 form solutions are obtained under the Poisson assumption as shown in Eq. (34), 374 which may overestimate the crossing rate. The comparison suggests that the closed375 form solutions are conservative in the cases studied here.

376 2. For the structural response peak factors calculated by the MCS that include the 377 intermittency effects are higher than those that disregard these effects. The likely 378 explanation is that, the PDF of the velocity increment at a small scale has larger 379 deviation than the Gaussian case, and accordingly the velocity increment has a 380 381 higher-rate of occurrence of the extremes. As the scales of these velocity increments overlap with the structural frequency, the structural response peak factor increases.

3. Generally, rigid structures are more sensitive to the intermittency effects as indicated by the comparison of MCS solutions. Intermittency being related to 
higher frequencies, i.e., velocity increments at smaller scales, tends to enhance excitation that are typical of relatively rigid structures. For the cases studied here, it is shown that the intermittency effects result in an average increase of $6.3 \%$ for the rigid structures (various combinations of the wind speeds and structural structures.

\section{CONCLUDING REMARKS}

391 A wavelet-based turbulent wind simulation scheme is developed in this study. This scheme

392 incorporates higher-order statistics of the turbulent wind fluctuations and is able to simulate

393 intermittency phenomenon. In traditional spectral representation or the time series models

394 based simulation with implied assumption of Gaussianity, the intermittency is masked, 395 therefore is not suitable for synthetic turbulence. Earlier examples of simulations based on 396 wavelet representations did not model necessary statistics of wavelet coefficients, whereas 397 the proposed scheme here incorporates these features. In addition, the effects of 398 intermittency on the wind-induced response based on the quasi-steady theory are assessed 399 for various cases. The results indicate that the intermittency tends to increase the extremes 400 of structural response, especially for relatively rigid structures. Also, structures of small 401 spatial dimensions will be more influenced by intermittency as these scales will engulf the 402 structures leading to enhanced loads. It is likely that intermittency may manifest more 403 energetic interactions with the vortical flow features around structures than those predicted 404 by the quasi-steady theory thus potentially leading to even higher extremes. 
406 The support for this project provided by the NSF Grant \# CMMI 15-37431 is gratefully

407 acknowledged.

\section{Appendix A. Determination of the Critical Scale}

409 Assume that the maximum point of the wavelet $\tilde{\psi}_{j}(f)$ portrays the representative

410 frequency of $\psi_{j}(t)$ at scale $j$. The critical scale $j_{c}$ corresponds to the wavelet whose

411 representative frequencies is closest to $f_{c}$. Figure 12 shows the Fourier spectra of several

412 normalized Haar wavelets.

\section{Appendix B. Relation between a Wavelet Coefficient and the Power Spectrum}

414 The correlation between two wavelet coefficients, $m_{j, k_{1}}$ and $m_{j, k_{2}}$, at the same scale $j$ is 415 denoted as $R\left(\zeta_{j, k_{1}}(\boldsymbol{\theta}) m_{j, k_{1}}, \zeta_{j, k_{2}}(\boldsymbol{\theta}) m_{j, k_{2}}\right)$. Let $V(t)$ be a stationary process, and then 416 express $R\left(\zeta_{j, k_{1}}(\boldsymbol{\theta}) m_{j, k_{1}}, \zeta_{j, k_{2}}(\boldsymbol{\theta}) m_{j, k_{2}}\right)$ further as follows

417

$$
\begin{aligned}
& R\left(\zeta_{j, k_{1}}(\boldsymbol{\theta}) m_{j, k_{1}}, \zeta_{j, k_{2}}(\boldsymbol{\theta}) m_{j, k_{2}}\right) \\
& =\left\langle\zeta_{j, k_{1}}(\boldsymbol{\theta}) m_{j, k_{1}}, \zeta_{j, k_{2}}^{*}(\boldsymbol{\theta}) m_{j, k_{2}}^{*}\right\rangle \\
& =\left\langle\int_{-\infty}^{\infty} \psi_{j, k_{1}}\left(t_{1}\right) V\left(t_{1}\right) d t_{1} \int_{-\infty}^{\infty} \psi_{j, k_{2}}^{*}\left(t_{2}\right) V^{*}\left(t_{2}\right) d t_{2}\right\rangle \\
& =\left\langle\int_{-\infty}^{\infty} \int_{-\infty}^{\infty} \psi_{j, k_{1}}\left(t_{1}\right) \psi_{j, k_{2}}^{*}\left(t_{2}\right) V\left(t_{1}\right) V^{*}\left(t_{2}\right) d t_{1} d t_{2}\right\rangle \\
& =\int_{-\infty}^{\infty} \int_{-\infty}^{\infty} \psi_{j, k_{1}}\left(t_{1}\right) \psi_{j, k_{2}}^{*}\left(t_{2}\right)\left\langle V\left(t_{1}\right) V^{*}\left(t_{2}\right)\right\rangle d t_{1} d t_{2} \\
& =\int_{-\infty}^{\infty} \int_{-\infty}^{\infty} \psi_{j, k_{1}}\left(t_{1}\right) \psi_{j, k_{2}}^{*}\left(t_{2}\right) R\left(V\left(t_{1}\right), V\left(t_{2}\right)\right) d t_{1} d t_{2} \\
& =\int_{-\infty}^{\infty} \int_{-\infty}^{\infty} \psi_{j, k_{1}}\left(t_{1}\right) \psi_{j, k_{2}}^{*}\left(t_{2}\right) \int_{-\infty}^{\infty} S_{V}(f) e^{i\left(t_{2}-t_{1}\right) 2 \pi f} d f d t_{1} d t_{2} \\
& =\int_{-\infty}^{\infty}\left\{\int_{-\infty}^{\infty}\left[\int_{-\infty}^{\infty} \psi_{j, k_{1}}\left(t_{1}\right) e^{-i t_{1} 2 \pi f} d t_{1}\right] \psi_{j, k_{2}}^{*}\left(t_{2}\right) e^{i t_{2} 2 \pi f} d t_{2}\right\} S_{V}(f) d f \\
& =\int_{-\infty}^{\infty} \tilde{\psi}_{j, k_{1}}(f) \tilde{\psi}_{j, k_{2}}^{*}(f) S_{V}(f) d f \\
& =2^{n-j+1} \int_{-\infty}^{\infty}|\tilde{\psi}(f)|^{2} e^{i 2 \pi f\left(k_{2}-k_{1}\right)} S_{V}(f) d f
\end{aligned}
$$


418 where the symbol '*' represents the conjugate counterpart; $R\left(V\left(t_{1}\right), V\left(t_{2}\right)\right)$ is the 419 correlation between $V\left(t_{1}\right)$ and $V\left(t_{2}\right)$. Since the final expression of $420 R\left(\zeta_{j, k_{1}}(\boldsymbol{\theta}) m_{j, k_{1}}, \zeta_{j, k_{2}}(\boldsymbol{\theta}) m_{j, k_{2}}\right)$ depends only the difference between $k_{1}$ and $k_{2}$, the

421 sequence $\left\{\zeta_{j, k}(\boldsymbol{\theta})\right\}_{k=1}^{2^{j-1}}$ is stationary. The scaling factor $m_{j, k}$ can be easily obtained as

$422 \quad m_{j, k}=\sqrt{2^{n-j+1} \int_{-\infty}^{\infty}|\tilde{\psi}(f)|^{2} S_{V}(f) d f}$

423 by setting $k_{1}=k_{2}$.

\section{REFERENCE}

425 [1] Arneodo, A., Bacry, E., \& Muzy, J. (1998). Random cascades on wavelet dyadic trees.

426 Journal of Mathematical Physics, 39(8), 4142-4164.

427 [2] Atta, C.V., \& Chen, W. (1970). Structure functions of turbulence in the atmospheric 428 boundary layer over the ocean. Journal of Fluid Mechanics, 44(01), 145-159.

429 [3] Bak, P., Tang, C., \& Wiesenfeld, K. (1988). Self-organized criticality. Physical review A, 38(1), 364-374.

[4] Basu, S., Foufoula-Georgiou, E., \& Porté-Agel, F. (2004). Synthetic turbulence, fractal interpolation, and large-eddy simulation. Physical Review E, 70(2), 026310.

[5] Castaing, B., Gagne, Y., \& Hopfinger, E. (1990). Velocity probability density functions of high Reynolds number turbulence. Physica D: Nonlinear Phenomena, 46(2), 177-200.

[6] Davenport, A.G. (1964). Note on the distribution of the largest value of a random function with application to gust loading. In ICE Proceedings, 28(2), 187-196. Thomas Telford.

[7] Frisch, U. (1995). Turbulence: the legacy of AN Kolmogorov. Cambridge university press.

[8] Ghanem, R.G., \& Spanos, P.D. (2003). Stochastic finite elements: a spectral approach. Courier Dover Publications.

[9] Grigoriu, M. (1986). Response of linear systems to quadratic Gaussian excitations. 
Journal of Engineering Mechanics, 112(6), 523-535.

445 [10] Gurley, K., \& Kareem, A. (1999). Applications of Wavelet Transforms in Wind, Earthquake and Ocean Engineering. Engineering Structures, 21(2), 149-167.

[11] Kareem, A., Tognarelli, M., \& Gurley, K. (1998). Modeling and analysis of quadratic term in the wind effects on structures. Journal of Wind Engineering and Industrial Aerodynamics, 74, 1101-1110.

[12] Kitagawa, T., \& Nomura, T. (2003). A wavelet-based method to generate artificial wind fluctuation data. Journal of Wind Engineering and Industrial Aerodynamics, 91(7), 943-964.

[13] Kolmogorov, A.N. (1941). The local structure of turbulence in incompressible viscous fluid for very large Reynolds numbers. In Dokl. Akad. Nauk SSSR, 30(4), 299-303.

[14] Kolmogorov, A.N. (1962). A refinement of previous hypotheses concerning the local structure of turbulence in a viscous incompressible fluid at high Reynolds number. Journal of Fluid Mechanics, 13(1), 82-85.

[15]Li, Y., \& Kareem, A. (1990a). ARMA representation of wind field. Journal of Wind Engineering and Industrial Aerodynamics, 36, 415-427.

[16]Li, Y., \& Kareem, A. (1990b). ARMA systems in wind engineering. Probabilistic Engineering Mechanics, 5(2), 49-59.

[17]Lutes, L.D., \& Sarkani, S. (1997). Stochastic analysis of structural and mechanical vibrations. Prentice Hall. Englewood Cliffs, NJ.

[18] Phoon, K., Huang, H., \& Quek, S. (2004). Comparison between Karhunen-Loeve and wavelet expansions for simulation of Gaussian processes. Computers \& Structures, 82(13), 985-991.

[19] She, Z.-S., \& Leveque, E. (1994). Universal scaling laws in fully developed turbulence. Physical Review Letters, 72(3), 336-339.

[20] She, Z.-S., \& Waymire, E.C. (1995). Quantized energy cascade and log-Poisson statistics in fully developed turbulence. Physical Review Letters, 74(2), 262-265.

[21] Shinozuka, M., \& Deodatis, G. (1991). Simulation of stochastic processes by spectral representation. Applied Mechanics Reviews, 44(4), 191-204.

[22] Soize, C. (1978). Gust loading factors with nonlinear pressure terms. Journal of the Structural Division, 104(6), 991-1007. 
475 [23] Sreenivasan, K.R., \& Antonia, R.A. (1997). The phenomenology of small-scale 476 turbulence. Annual Review of Fluid Mechanics, 29(1), 435-472.

477 
478 Figure 1: PDFs of the normalized velocity increments of a wind velocity process in various

479 time intervals.

480

481 Figure 2: Different Wavelet bases. (a) Haar mother wavelet; (b) Meyer mother wavelet.

483 Figure 3: Relations between the Haar wavelet coefficients and velocity increments.

$485 \quad$ Figure 4: The simulation procedure of turbulent wind.

487 Figure 5: Measurement and simulation of wind processes.

$489 \quad$ Figure 6: Power spectrum.

Figure 7: PDF of wavelet coefficients at various scales in the inertial sub-range.

493 Figure 8: The present simulation scheme in the inertial sub-range.

$495 \quad$ Figure 9: The simulation scheme of wavelet dyadic tree.

497 Figure 10: The distribution configuration of samples. Different symbols denote different 498 realizations. (a) Samples of $X$ and $\left\{Y_{i}\right\}_{i=1}^{20}$ to simulate $Z_{1}$; (b) Samples of $X$ and $\left\{\tilde{Y}_{i}\right\}_{i=1}^{20}$ to 499 simulate $Z_{2}$.

501 Figure 11: The simulation scheme of the modification model.

503 Figure 12: Fourier spectra of several Haar wavelets. The bold dash line represents the 504 representative frequency of the wavelet at the scale $j=1$. 


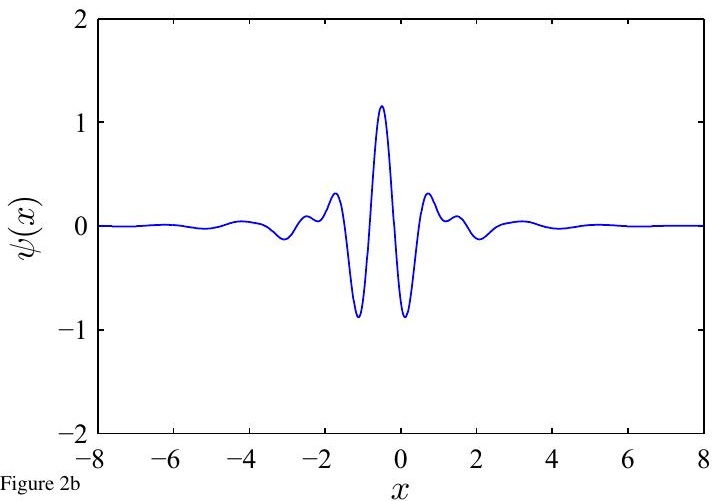




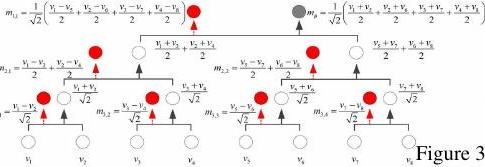




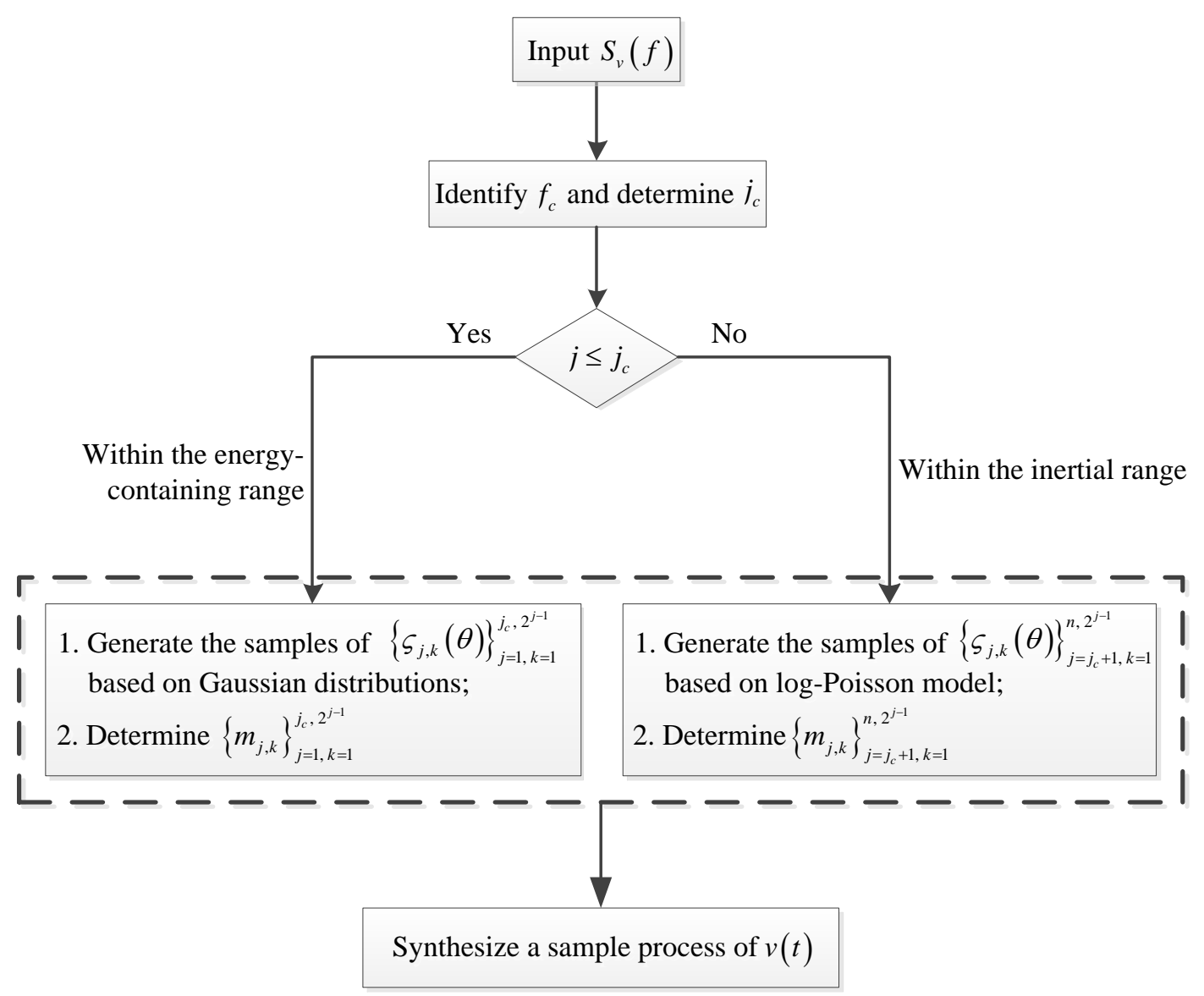




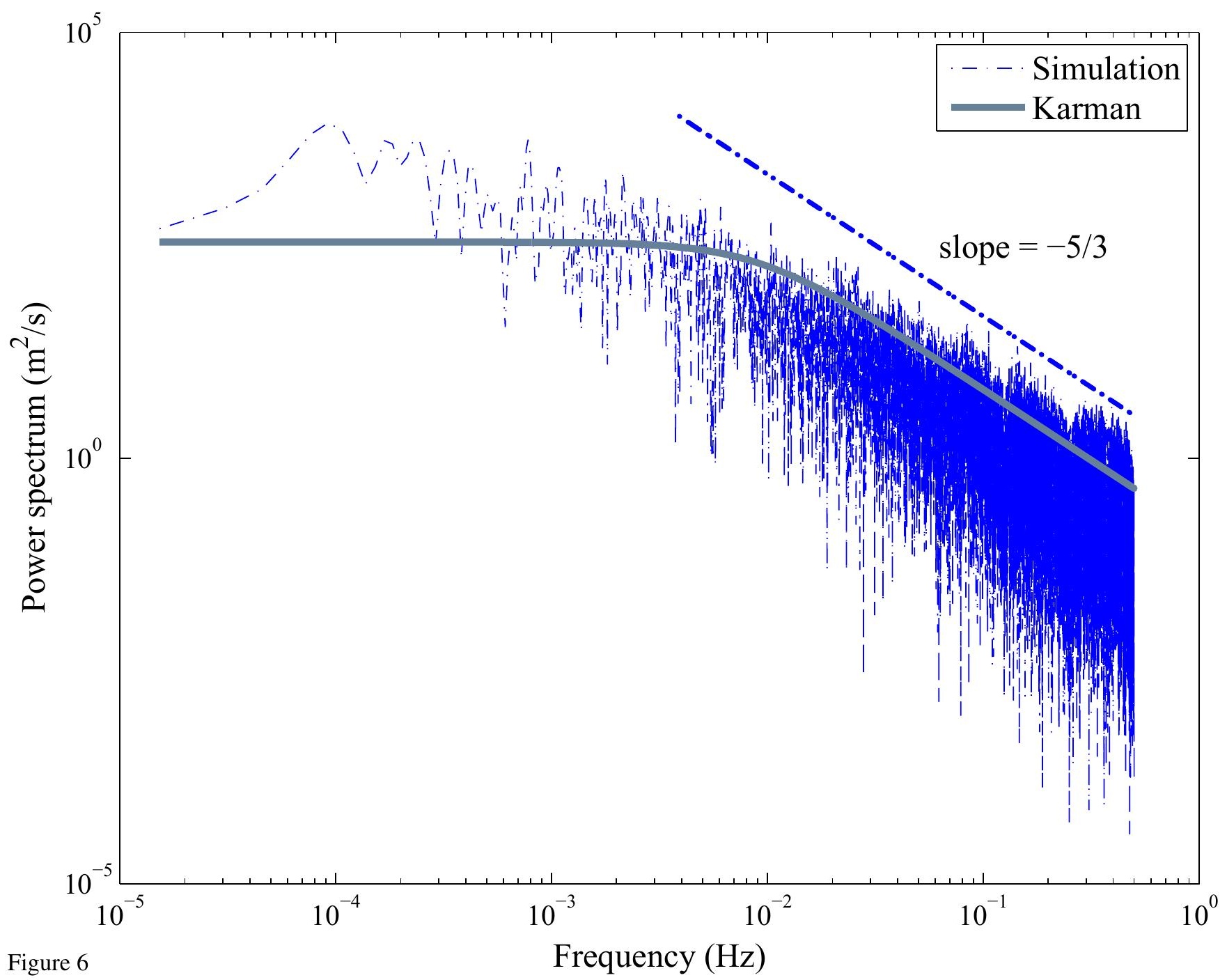




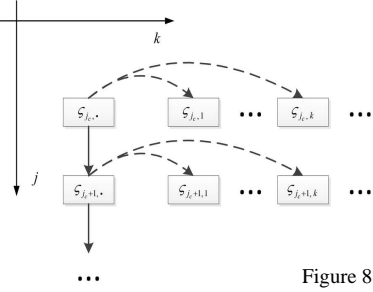




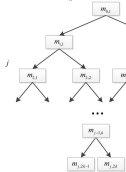

n 


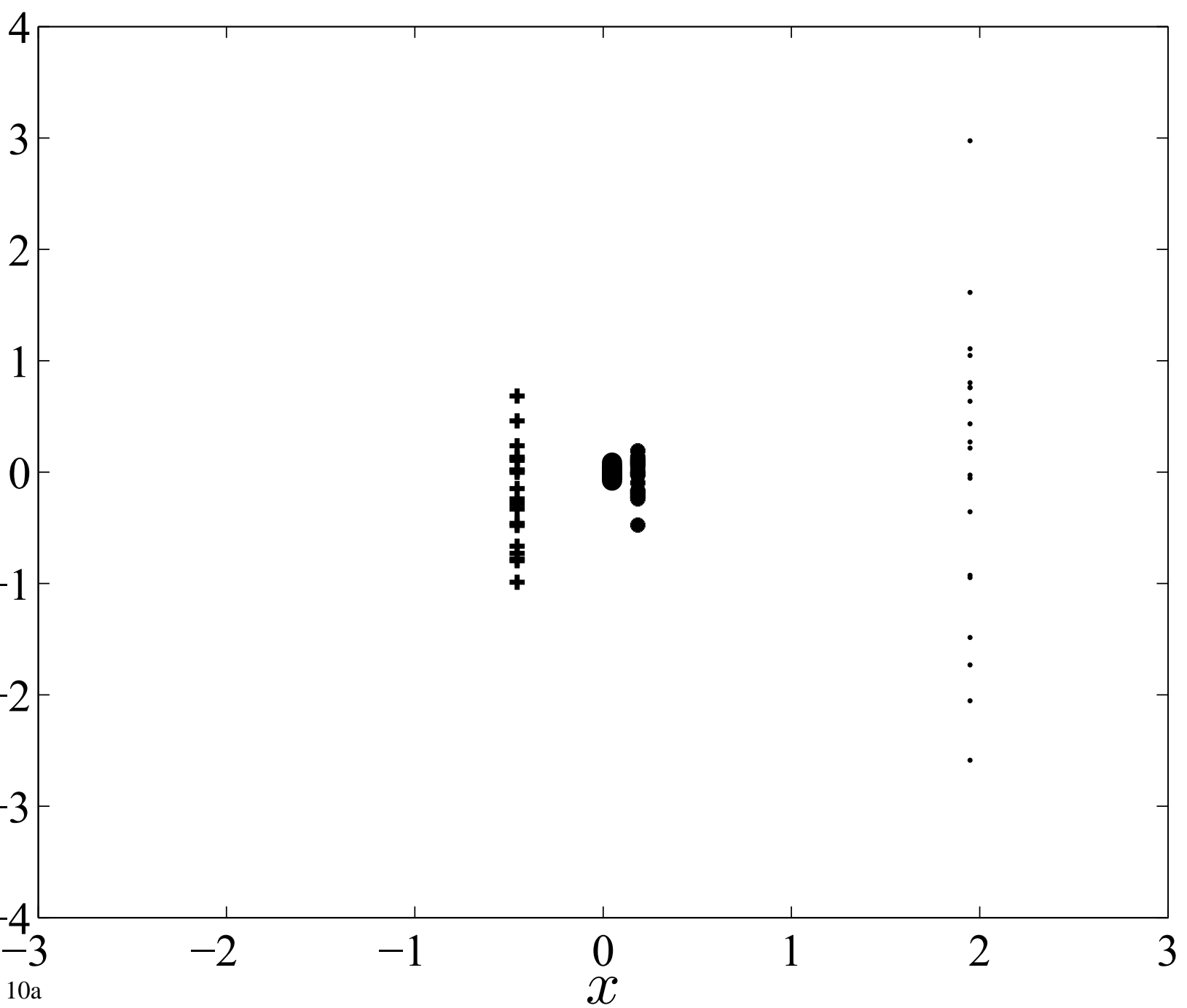




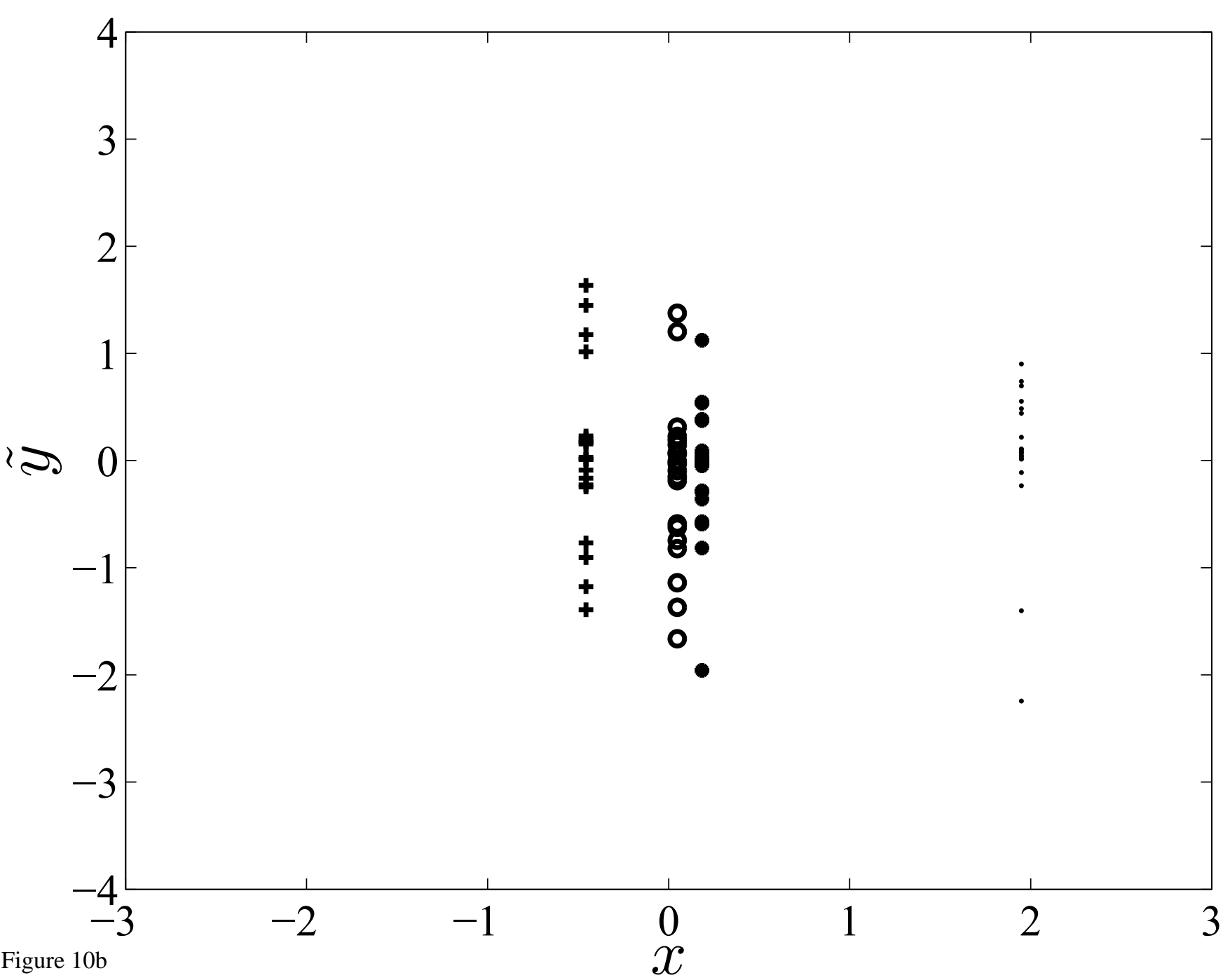



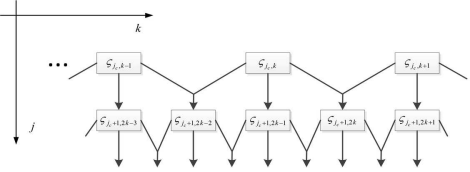

Figure 11 


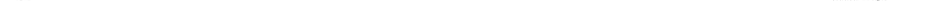


Table 1: Comparison of structural response peak factors

\begin{tabular}{|c|c|c|c|c|c|c|c|c|c|c|}
\hline \multirow[b]{3}{*}{ Case } & \multirow{3}{*}{$\begin{array}{l}\text { Reference } \\
\text { wind } \\
(\mathrm{m} / \mathrm{s})\end{array}$} & \multirow{3}{*}{$\begin{array}{l}\text { Power } \\
\text { law } \\
\text { exponent }\end{array}$} & \multirow{3}{*}{$\begin{array}{l}\text { RMS } \\
(\mathrm{m} / \mathrm{s})\end{array}$} & \multirow{3}{*}{$\begin{array}{l}\text { Damping } \\
\text { ratio } \\
(\%)\end{array}$} & \multirow{3}{*}{$\begin{array}{c}g \\
G-G \\
\text { Davenport }\end{array}$} & \multicolumn{5}{|c|}{$g_{n g}$} \\
\hline & & & & & & \multicolumn{4}{|c|}{$G-N G$} & \multirow{2}{*}{$\begin{array}{c}N G-N G \\
\text { MCS (Present study) }\end{array}$} \\
\hline & & & & & & Soize & Grigoriu & Kareem & MCS & \\
\hline \multicolumn{11}{|c|}{$h=20 \mathrm{~m}, M=2161 \mathrm{~kg}, K=85300 \mathrm{~N} / \mathrm{m}$} \\
\hline 1 & 28 & 0.15 & 4.6 & 5 & 4.04 & 4.64 & 5.18 & 5.09 & 4.81 & 5.33 \\
\hline 2 & 13 & 0.35 & 5.8 & 3 & 4.10 & 4.91 & 6.43 & 6.50 & 6.01 & 6.18 \\
\hline 3 & 13 & 0.35 & 5.8 & 5 & 4.04 & 4.86 & 6.42 & 6.44 & 6.03 & 6.41 \\
\hline \multicolumn{11}{|c|}{$h=80 \mathrm{~m}, M=13500 \mathrm{~kg}, K=85300 \mathrm{~N} / \mathrm{m}$} \\
\hline 4 & 28 & 0.15 & 4.6 & 3 & 3.87 & 4.29 & 4.59 & 4.49 & 4.38 & 4.62 \\
\hline 5 & 28 & 0.15 & 4.6 & 5 & 3.85 & 4.31 & 4.63 & 4.56 & 4.44 & 4.81 \\
\hline 6 & 13 & 0.35 & 5.8 & 3 & 3.87 & 4.43 & 5.13 & 5.01 & 4.83 & 4.90 \\
\hline 7 & 13 & 0.35 & 5.8 & 5 & 3.85 & 4.45 & 5.12 & 5.14 & 4.95 & 5.13 \\
\hline
\end{tabular}

Notation:

RMS: root mean square wind velocity;

$g$ : Gaussian structural response peak factor;

$g_{n g}$ : non-Gaussian structural response peak factor;

$G-G$ : Gaussian wind velocity process and Gaussian structural response peak factor;

$G-N G$ : Gaussian wind velocity process and non-Gaussian structural response peak factor;

$N G-N G$ : turbulent wind velocity process and non-Gaussian structural peak factor;

Davenport: a closed-form solution (Davenport, 1964);

Soize: a closed-form solution(Soize, 1978);

Grigoriu: a closed-form solution(Grigoriu, 1986);

Kareem: a closed-form solution(Kareem et al., 1998). 